\title{
Competencias del Docente Deportivo y Actitud de Servicio en Instituciones Educativas
}

\section{Competences of the Sports Teacher and Attitude of Service in Educational Institutions}

INFORMACIÓN DEL

\section{ARTÍCULO}

Fecha de recepción: 29 de Agosto de 2019.

Fecha de aceptación: 3 de Diciembre de 2019.

${ }^{1}$ Maestría en Ciencias de la Educación, Universidad Dr. Rafael Belloso Chacin. Investigador de la Universidad Dr. Rafael Belloso Chacin - Venezuela. E-mail: larrycallesv@gmail.com

Código ORCID

https://orcid.org/0000-0002-9286-0635

2 Doctor en Ciencias de la Educación, Universidad Dr. Rafael Belloso Chacin. Docente e investigador de la Universidad de Pamplona - Colombia. E-mail: kleeder8@hotmail.com

Código ORCID:

https://orcid.org/0000-0003-3644-0561

CITACIÓN: Calles Vence , L. J., \& Bracho Pérez, K. J. (2019). Competencias del Docente Deportivo y Actitud de Servicio en Instituciones Educativas. PODIUM, (36), 55-72. https://doi.org/10.31095/podium.2019.36.4

\section{ENLACE DOI:}

http://dx.doi.org/10.31095/podium.201 9.36 .4

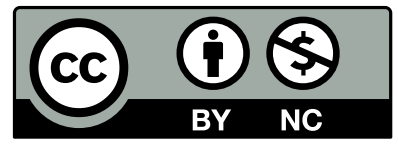

Larry Jhon Calles Vence ${ }^{1}$, Kleeder José Bracho Pérez ${ }^{2}$

\section{Resumen}

El propósito del estudio fue determinar la relación entre competencias del docente deportivo y su actitud de servicio. La investigación fue descriptiva y no experimental. A la población representada por 24 directivos y 26 docentes de Instituciones Educativas Primarias Bolivarianas del Municipio Mara, Estado Zulia, se le aplicó un instrumento de preguntas cerradas de 39 ítems, validado por cinco expertos, cuyo coeficiente alfa cronbach fue de 0,92 . Se realizó el análisis estadístico descriptivo de los datos, permitiendo el análisis y discusión por dimensiones e indicadores. Directivos y docentes afirman que no van más allá de lo que plantean las programaciones de trabajo, escasamente mantienen compromisos con el desarrollo organizacional y reflexionan poco sobre las consecuencias de los actos que realizan.

\section{Palabras Clave: \\ Actitud, Competencias, Docente, Deporte,Servicio, Venezuela.}

Clasificación JEL: I210.

\begin{abstract}
The aim of the article was to determine the relationship between sports teacher competencies and their service attitude. The research was descriptive and not experimental. To the population represented by 24 executives and 26 teachers of Instituciones Educativas Primarias Bolivarianas del Municipio Mara, Estado Zulia, a 39 items questionnaire (alpha cronbach coefficient $=0.92$ ) was applied, previously it was validated by five experts. The descriptive statistical analysis of the data was carried out, allowing the analysis and discussion by dimensions and indicators. Managers and teachers affirm that they do not go beyond what work schedules suggest, they scarcely maintain commitments to organizational development and reflect little on the consequences of the acts they perform.
\end{abstract}

Keywords:

Attitude, Competencies, Teacher, Sport, Service, Venezuela.

JEL Classification: I210. 


\section{Introducción}

A nivel mundial la educación ha venido realizando cambios a nivel de contenidos como de estrategias, las cuales toman en cuenta el desenvolvimiento de las labores docentes, ésto debido a la actitud que tienen los educadores sobre el proceso educativo generado dentro de las aulas de clases o canchas deportivas. Es necesario resaltar, que dichos cambios se generan por las competencias y habilidades que tiene el docente en el desarrollo de sus actividades, en el caso más puntual, cuando se trata de la educacion deportiva. Según Torres (2009), los docentes que se desenvuelven en las áreas deportivas dejan de tener competencias específicas y generales lo que conlleva a que la actitud de servicio sea basada en los elementos básicos como la vocacion, compromiso o simplemente se tomen actitudes de apatía y conflicto frente a la necesidad imperante de desarrollar competencias tales como de salud física, mental o de promotor social lo cual permitiría generar eficiencia en la praxis pedagógica. En vista de lo planteado, el docente deportivo tiene la necesidad de ser un profesional proactivo alejando la apatía y optimista alejando los conflictos en las aulas o canchas deportivas.

Ahora bien, en las instituciones Latinoamericanas de los países como Colombia, Perú, Chile y Argentina, los docentes deportivos han demostrado desinterés o carencias en el manejo de sus competencias, simplementes están en la labor por asuntos monetarios, olvidando la importancia que tiene el desarrollo de competencias gerenciales y especificas, los cuales hacen del docente una persona proactiva, demostrando una actitud optimísta hacia el servicio que ofrece en el área deportiva educativa (González, 2010).

El desarrollo del deporte en las instituciones es de suma importancia, ya que ayuda a los estudiantes a mantener activo el proceso psicomotor, específicamente cuando se integran los movimientos corporales y el conocimiento. Bajo esta perspectiva Isaza (2010), señala que los docentes deportivos son aquellos que favorecen la búsqueda del desarrollo eficaz $\mathrm{y}$ consciente del movimiento corporal en la interacción del joven con el medio en el cual se desenvuelve, a través de la manifestación de actividades de integración social. En otras palabras, son las personas que se encargan de llevar actividades donde exista la interacción de la comunidad con el ambito educativo. Con base en lo anteriormente planteado, en Venezuela según el Plan de la Patria (2013-2019) presentado por Maduro (2013), en las instituciones educativas se debe propiciar las condiciones para el desarrollo de una cultura de recreación y práctica deportiva liberadora, ambientalista e integradora en torno a los valores de la Patria, como vía para la liberación de la conciencia, la paz y la convivencia armónica. En el cual, en su mayoria las instituciones han acatado lo establecido por el plan a pesar de que puedan existir debilidades en el manejo de las competencias y actitud hacia el servicio. Bracho (2019), señala que es necesario que el docente se apropie de 
competencias que vayan más allá de su actividad rutinaria en el aula, que sea capaz de afrontar la realidad que vive, a fin de dar viabilidad a los problemas que enfrenta desde su práctica pedagógica.

Por su parte, los docentes deportivos serían las personas encargadas de propiciar las condiciones para el desarrollo de una cultura de recreación y práctica deportiva liberadora, a través de la planificación constante de actividades deportivas, donde se involucren no sólo a los estudiates de las instituciones educativas sino que también se puedan involucrar a las comunidades, sirviendo como promotor social. Esta competencia estaría basada en investigación, lo cual contribuiría a que exista adecuada praxis pedagógica, es decir, que el docente maneje herramientas $y$ estrategias adecuadas para llevar el conocimiento logrando al aprendizaje significativo. Asimismo, el docente es un investigador que a menudo maneja contenidos que debe reforzar incluyendo todas ese competencias que posee, involucrando a todas las personas con las que quiere contar en la generación de conocimientos propios. En muchas ocasiones, el desconocimiento de las comptencias gerenciales y específicas hace que el docente mantenga una actitud negativa hacia el servicio que presta como educador, no sólo desde su perspectiva, sino también desde la perspectiva del estudiante (Alles, 2009).

Tomando en consideración a Morales (2007, p. 87), se hace necesario señalar que "el trabajo lo podemos definir como la manera de proyectarnos a los demás".
En otras palabras, que los docentes han perdido, según la misma autora, la vocación de servicio, simplemente se ajustan a un esquema salarial, olvidando el compromiso y la capacidad de asistencias que deben tener hacia los estudiantes. Por su parte, en las Instituciones de Educación Primaria Bolivariana del Municipio Mara del Estado Zulia, quizás existen deficiencias en las competencias específicas que manejan los docentes deportivos, lo que pudiese incidir en su actitud de servicio hacia los estudiantes. Ahora bien, según observaciones no sistematizadas se puede señalar que el docente deportivo desarrolla actividades físico-motoras llevando a los estudiantes a mantener un estilo de vida saludable, manteniéndolo en movimientos que le ayudan a cada uno de ellos a mantener los músculos de su cuerpo.

De igual forma, el docente deportivo pareciera tener la necesidad del manejo de competencias, ya sean gerenciales como también específicas, debido a que en la actualidad se carece de conocimiento en elementos que tienen que ver con la salud física y mental para que sean aplicados a los estudiantes, dándoles a entender la importancia que tiene el desarrollo de la actividad deportiva en el crecimiento como individuo. El conocimiento sobre la importancia de la salud física y mental permite que exísta el equilibrio emocional de las personas. El docente deportivo para Morales (2007), al carecer de esas competencias específicas o gerenciales llevaría como consecuencias la necesidad de una eficiente praxis 
pedagógica lo que ocasionaría que dejaran de ser promotores sociales, es decir se encontrarían en desventajas para que lleven a las comunidades la informacion necesaria para el crecimiento deportivo.

De continuar la situación antes descrita, los docentes fijarían su postura en una actitud apática y conflictiva demostrando posiblemente desapego en los procesos deportivos o simplemente alejamiento de su rol como docentes de educación física. Esas actitudes provocan que el docente deje de ser proactivo y optimista, lo que genera que el estudiante se sienta desmotivado por las actividades deportivas, lo cual, generaría desacuerdos entre las actividades planteadas y el apoyo de los estudiantes, ya que se sentirían desinterados en cumplir el proceso deportivo. Por su parte, el docente seguiría en la necesidad de vocación, ya que sólo se adecua a un incentivo salarial dejando de explotar sus competencias.

Para poder atacar la situación, sólo se requiere determinar la relación que existe entre las competencias del docente deportivo y su actitud de servicio, basado específicamente en identificar las competencias generales como: Salud física, salud mental y el equilibrio emocional, describir las competencias específicas de la praxis pedagógica, como promotor social y de investigador, además de caracterizar los elementos básicos y definir el tipo de actitud de servicio, permitiendo, establecer la relación entre competencias del docente deportivo y su actitud de servicio en Instituciones de Educación Primaria Bolivariana.

\section{Revisión de literatura}

\section{Competencias del Docente Deportivo}

Las competencias son unas características subyacentes en el individuo, relacionada con un estándar de efectividad o desempeño superior en un trabajo o situación determinados. Es necesario resaltar que las competencias del docente deportivo se basan en las diferentes habilidades que demuestran la capacidad de gerencia y autocontrol de los contenidos ofrecidos. Al respecto, Pérez y Camejo (2009, p. 152), define las competencias del docente deportivo como "el conjunto de rasgos que caracterizan al profesional de la educación que ejerce el cargo de inspector de las actividades escolares". La relevancia de las competencias es que constituye un modelo a seguir en el proceso y formación en el desempeño de la docencia, y este se fundamenta en su comportamiento como persona integral, dotado de competencias.

\section{Competencias Generales}

Existe una necesidad creciente de aplicar una gerencia efectiva en las organizaciones educativas a fin de corregir de manera acertada su praxis. La gestión en una institución educativa consiste en aplicar las competencias generales y específicas para determinar y lograr los objetivos propuestos. Ahora bien, Tobón (2005, p. 54), señala que las competencias generales "son una especialización del impacto e influencia, y reflejan el interés por causar un efecto concreto para que las personas dirijan su 
actividad en un sentido concreto". Basado en lo anterior, el docente deportivo debe estar vinculado con todas las funciones y procesos de la institución, con la finalidad de conocer las características y cualidades que tiene cada uno en el desarrollo de las actividades permitiendo vincular a los estudiantes con cada una de las actividades, tareas y acciones.

\section{Competencias Físicas}

En las instituciones educativas los docentes deportivos deben tener competencias físicas para el desarrollo de las actividades académicas. El desarrollo psicomotor de los estudiantes es una de las piezas claves en el proceso educativo, de allí la importancia de que el docente se encuentre en la capacidad de preparar a los estudiantes realizando tareas deportivas que generen salud y seguridad. Álvarez (1985, citado por Tobón 2005, p. 24) define las competencias físicas como "los factores que determinan la condición física de un individuo y lo orientan para la realización de una determinada actividad física, posibilitando mediante el entrenamiento que un sujeto desarrolle al máximo su potencial físico". De allí el docente educativo debe tener la capacidad, habilidad y destreza para desarrollar actividades dentro y fuera de las aulas de clases, tales como juegos deportivos, calentamientos, entre otros.

\section{Capacidades Mentales}

Los docentes deportivos deben tener competencias mentales para enseñar a los estudiantes el proceso deportivo. Al respecto, Sanz (2010, p. 22), señala que "las competencias mentales tienen como finalidad preferente comprender, evaluar y generar información, tomar decisiones y solucionar problemas", es decir que los docentes manejan conocimientos específicos que les permite involucrar al docente deportivo y a los estudiantes de las instituciones educativas para poder realizar efectivas tomas de decisiones. Asimismo, esa toma de decisión permite el efectivo desarrollo de las actividades deportivas dentro y fuera de las instituciones.

\section{Capacidades Emocionales}

Bunk (1994, p. 9, citado por Robbins y De Cenzo 2009, p. 129), señala que "las competencias emocionales son un conjunto de conocimientos, destrezas y aptitudes necesarias para ejercer una profesión, resolver problemas profesionales de forma autónoma y flexible, y ser capaz de colaborar con el entorno de la organización". Es necesario relacionar no sólo al estudiante con las competencias del docente deportivo, sino que también es necesario relacionarlo con el entorno ya que este ayuda a ajustar las actividades.

\section{Competencias Especificas}

Para Alles (2009, p. 234), las competencias específicas "son necesarias en aquellas personas a cargo y sus posiciones son de jefatura o gerencia". En otras palabras, el docente gerencia en el aula de clases todas las actividades tomando en consideración la satisfacción, motivación y otros elementos propios de la profesión, lo cual permite el desenvolvimiento y logro de los objetivos planteados. Dentro de las 
competencias específicas se toma en consideración la praxis pedagógica (la forma como se desarrolla la labor).

\section{Praxis Pedagógica}

La praxis pedagógica de los docentes deportivos está referida a un proceso que se cumple en todas las actividades que se desenvuelve en las instituciones educativas, evaluando los resultados con la finalidad de cuantificar y calificar los logros obtenidos en base a las metas propuestas, así como determinar las acciones por ejecutar, para llegar a ello, a través de la escogencia e implementación de nuevas estrategias para alcanzarlos. Por su parte, Vezub y Alliaud (2012), señala que la praxis pedagógica está referida en la Educación, y por consecuencia a las ciencias de la educación.

\section{Promotor Social}

El docente deportivo es un promotor social en la medida en que se desarrollan las actividades permitiendo que exista el involucramiento de todas las partes (estudiantes, comunidad, docentes, entre otros), que forman la comunidad educativa. Por su parte, Oliver, López y Climent (2010, p. 89), señalan que el docente promotor social "es aquella persona que hace que el estudiante tenga la oportunidad de desarrollarse como líder ante la comunidad y la escuela, lo cual lo prepara para afrontar su futuro inmediato como profesional de la educación".

\section{Investigador}

El docente deportivo en su rol de investigador debe asumir una actitud hacia su profesionalización, para así desenvolver conocimientos que interfieran en su experiencia laboral y pueda conducir con propiedad el desarrollo integral. En la educación, la investigación demanda competencias científicas específicas, lo que representa que están autorizados para tomar decisiones sobre la praxis educativa y políticas. Para Chiavenato (2006 p. 250), la función investigadora "es un procedimiento ordenado controlado y riguroso, mediante el cual manipulamos objetos, hechos o ideas para corregir o verificar el conocimiento en cualquiera de los dominios de la ciencia y de la educación". Se busca que el docente deportivo tome la investigación como un mecanismo para resolver las dificultades cotidianas.

\section{Actitud de Servicio}

Summers (2006, p. 34) señala que la actitud de servicio "es una predisposición a responder a un objeto determinado, y no la conducta efectiva hacia él". Basado en lo anterior, la disposición es una de las cualidades características de la actitud, por lo que se explica más por la vía procesual que por la vía del producto. Es necesario entender que la actitud de servicio permite que el docente deportivo desarrolle las actividades mostrando interés y siendo promotor social de esas actividades deportivas.

\section{Elementos Básicos}

Para el desarrollo de las actividades deportivas, dentro de las instituciones educativas, es necesario tomar en consideración los elementos básicos de la 
actitud de servicio que ejerce el docente, mostrando cada una de las capacidades y habilidades para servir no sólo a los estudiantes sino a la comunidad educativa. Olson y Zanna (1993 citado por Myers 2005, p. 87), señala que los elementos básicos de la actitud de servicio "son consideradas, en conjunto, como las reacciones evaluativas favorables e inconvenientes hacia algo, reflejadas en creencias, sentimientos o inclinaciones a actuar de una persona".

\section{Tipos de Actitud}

Respecto a los tipos de actitud, estas han sido clasificadas de diversa forma. Bohlander, Snell, y Morris (2017, p. 67) sugiere que la actitud neutra "es aquella susceptible a modificaciones, es decir, puede transformarse en positiva o negativa". Los docentes deportivos son las personas que evidencian actitud neutral, ya que tienden a descubrir, así como observar al objeto actitudinal, no relacionado consigo mismo, sin rechazarlo o favorecerlo. Esta actitud no implica un grado de favorabilidad o desacuerdo con respecto a la evaluación o predisposición determinada por el objeto actitudinal.

\section{Proactiva}

Una estrategia educativa calificada como proactiva debe clarificar de forma realista dónde está la institución, porqué está donde está, a dónde quiere ir, y formular cómo revisar posteriormente la estrategia de manera sistemática y proactiva. Esta amplia batería de preguntas existenciales constituye el corazón del proceso estratégico.
Edvinsson y Malone (2007, p. 54), señalan que:

La realidad institucional tiende a ser proactiva por excelencia, sólo cuando la dirección llega a la etapa del desencanto; cuando los contactos que llegan no son los mejores; cuando los educadores prefieren hacer cualquier otra tarea antes que ponerse a educar; las llamadas telefónicas son más bien de estudiantes que de interesados $\mathrm{y}$ las llamadas entrantes y las solicitudes de información no generan suficientes estudiantes potenciales para garantizar un crecimiento sostenido de la institución, se plantea la formulación de una estrategia de crecimiento.

\section{Optimista}

El docente optimista busca siempre dar todos los conocimientos a los estudiantes, así como lo plantea Edvinsson y Malone (2007, p. 76), "es una actitud que impide caer en la apatía, la desesperación o la depresión frente a las adversidades. La noción de optimismo se opone al concepto filosófico de pesimismo". En otras palabras, siempre hay alternativas de solución, donde el docente conduce a sus estudiantes a lograr sus metas académicas, formándolos para el campo laboral.

\section{Apática}

Los docentes muchas veces demuestran actitudes apáticas frente a sus labores, por 
razones ya sean personales $\mathrm{o}$ profesionales, tales como factores familiares, económicos, sociales, vocacionales, entre otros, que hacen que se pongan ajenos o desinteresados en el cumplimiento de su labor deportiva. En este sentido, la conducta requiere de carácter cognitivo para que pueda desarrollar cada uno de los elementos de la actitud que según Reinfeld y Rojas (2005, p. 12):

Es una emoción que se manifiesta como todas, en varios aspectos: cognitivos, conductuales $\mathrm{y}$ afectivos con su correspondiente correlato neuroquímico $\mathrm{y}$ anatómico. La disminución de la motivación (su expresión conductual), es lo que genera la confusión con la depresión, ya que la apatía y la depresión la presentan de manera muy marcada, la depresión en la mayoría de los casos y en la apatía, siempre.

\section{Conflictiva}

Ramírez (2002, citado por López, Montes y Vázquez 2007, p. 98), señala que con las conductas conflictivas del docente "se determinan factores estructurales siendo parte integral de los procesos de cambio, por lo que es parte natural de cualquier relación comunicacional". Sin embargo, lo importante es saber cómo manejarlo, puesto que puede tener diversas consecuencias dentro de la institución educativa. El éxito de las organizaciones se funde en cómo superan sus conflictos.

\section{Metodología}

El estudio se encontró asentado bajo el paradigma positivista. Según Chávez (2007, p.28), "esta tendencia sigue un enfoque que orienta al método empírico experimental. Sostiene que fuera del ser humano no existe una realidad social externa y objetiva ya concebida. $\mathrm{Su}$ objetivo se basa en manifestar la realidad existente $\sin$ modificarla". En este sentido, se discurre según el procedimiento utilizado como una investigación de tipo descriptivo y correlacional, ya que el designio es describir hechos y situaciones. De acuerdo a Bavaresco (2008, p.26) "la investigación descriptiva consiste en describir sistemáticamente características homogéneas de los fenómenos estudiados sobre la realidad". Ahora bien, con esta premisa la investigación se orienta a discutir los datos relacionados con el estado real del contexto donde ocurren los hechos a explorar.

Chávez (2007) también establece que la investigación se caracteriza por ser correlacional: "Los estudios correlaciónales están dirigidos a determinar el grado de relación entre variables, manifestando el nivel en que una depende de la otra" (p. 185), es decir, se pretende establecer qué nivel de relación se asocian las competencias del docente deportivo y su actitud de servicio en un tiempo y lugar determinado.

En cuanto al diseño de la investigación, es no experimental, transversal, de campo. Hernández Fernández y Baptista (2008), expresan 
que los estudios no experimentales, se efectúan sin manipular intencionalmente las variables, es decir, se observar el fenómeno tal y como se dan en su contexto habitual, para ulteriormente ser analizado. Desde la perspectiva de Vieytes (2004), afirma que "los estudios transversales cristalizan los datos recogidos en un tiempo único", (p.119), es decir, se recolectan en un solo momento, desde este punto de vista la información suministrada por los sujetos se hace en un tiempo único, con el propósito de describir la variable, así como estudiar la incidencia, ocurrencia e interrelación en un instante dado.

Prosiguiendo con lo antes expuesto, la investigación es de campo, debido a que debe señalarse que se comprueban los hechos en un contexto establecido. Este marco de ideas es sustentado por los aportes de Arias (2004), quien señala que la exploración de campo consiste en recaudar los datos directamente de los investigados en su contexto y bajo la realidad donde acontecen las situaciones sin sesgar las variables. Resulta claro que se detecta indiscutiblemente el comportamiento de los sujetos en el entorno donde acontecen los hechos.

En la Tabla 1 se detalla la población objeto de estudio de las instituciones de educación primaria del Municipio Mara.

Para el levantamiento de datos se utilizó un cuestionario, versionado para Personal Directivo y Docentes de Educación Física estructurado en tres partes: 1) Presentación del aval de la institución que respalda el estudio y a quién va dirigido, 2) instrucciones generales para la explicación $y, 3)$ los 36 reactivos, los cuales midieron las variables con sus respectivas dimensiones e indicadores, utilizando una escala Likert con las alternativas: siempre (4), casi siempre (3), casi nunca (2), y nunca (1).

El instrumento se sometió a la validez

Tabla 1.

Instituciones Educativas del Municipio Mara: Población de estudio

\begin{tabular}{lccc}
\hline $\begin{array}{c}\text { Instituciones de Educación } \\
\text { Primaria Bolivariana }\end{array}$ & $\begin{array}{c}\text { Personal } \\
\text { Directivo }\end{array}$ & Docentes Deportivo & Total \\
\hline E.B.N. Francisco Araujo & 3 & 3 & 6 \\
E.B.N. Los Vaqu iros & 2 & 2 & 4 \\
E.B.N. Monte Verde & 2 & 2 & 4 \\
E.B.N. Brisas de Mara & 2 & 2 & 4 \\
E.B.N. Miriam Rubio & 2 & 2 & 4 \\
E.B.N. José Ángel Huerta & 2 & 2 & 4 \\
E.B.N. Leonor de Fernández & 3 & 4 & 7 \\
E.B.N. 5 de Julio & 2 & 2 & 4 \\
E.B.N. Guareira & 2 & 2 & 4 \\
E.B.E. San Andrés & 1 & 1 & 2 \\
E.B.E. Las Cruces & 3 & 4 & 7 \\
\hline \multicolumn{1}{c}{ Total } & 24 & 26 & 50 \\
\hline
\end{tabular}

Fuente: Calles y Bracho (2019). 
de contenido, mediante el juicio de cinco expertos. Se realizó una prueba piloto en una institución con características similares a la población objeto de estudio, y se aplicó la prueba del coeficiente Alfa de Cronbach, el cual determinó la confiabilidad del cuestionario, obteniendo el resultado de $r=0,92$.

Para el análisis de los datos, se aplicó estadística descriptiva (tablas de frecuencias y cálculo de medidas de tendencia central). Para el análisis se empleó un baremo (Tabla 2).

Tabla 2.

Baremo de Ponderación para la Categorización de Resultados sectoriales

\begin{tabular}{cc}
\hline Categoría & Rango \\
\hline Muy Adecuada & $3,41 \leq \mathrm{x} \leq 4,00$ \\
Adecuada & $2,81 \leq \mathrm{x} \leq 3,40$ \\
Medianamente Adecuada & $2,21 \leq \mathrm{x}<2,80$ \\
Poco Adecuada & $1,61 \leq \mathrm{x}<2,20$ \\
Nada Adecuada & $1,00 \leq \mathrm{x}<1,60$ \\
\hline Fuente: Calles y
\end{tabular}

\section{Resultados}

La variable competencias del docente deportivo que muestra la Tabla 3 , refleja la actuación de esta variable por dimensión de la siguiente manera: la

Tabla 3.

Competencias del Docente Deportivo dimensión competencias generales, se exhibe con un $37,2 \%$, para los directivos en la alternativa casi nunca, seguido de un $33,3 \%$, que se situaron en la alternativa siempre, mientras que el $27,1 \%$, se situaron en la alternativa nunca, quedando un $15,2 \%$, en la alternativa casi siempre. Se puede evidenciar que la medida de tendencia central para esta dimensión por parte de los directivos se situó en un 1.49, categorizándose según el baremo como nada desarrollado. Conforme a las respuestas suministradas por los docentes, se muestra que un $40,5 \%$, se situaron en la alternativa siempre, seguido de un $37,4 \%$, que se ubicaron en la alternativa casi siempre, mientras que el $7,1 \%$ se ubica en la alternativa nunca y solo el $2,2 \%$ se situaron en la alternativa casi nunca. Es de destacar, que se puede evidenciar que la medida de tendencia central para esta por parte de los sujetos sometidos al estudio se situó en un 1.71, categorizándose según el baremo como poco desarrollado.

Seguidamente, los porcentajes logrados en la dimensión competencias específicas, revela que un $22,6 \%$, de los directivos se situaron en la alternativa casi siempre, seguido de un $12,0 \%$, que se

\begin{tabular}{|c|c|c|c|c|c|c|c|c|c|c|}
\hline \multirow{3}{*}{ Dimensiones } & \multicolumn{8}{|c|}{ Alternativas (\%) } & \multirow{2}{*}{\multicolumn{2}{|c|}{ Medias }} \\
\hline & \multicolumn{2}{|c|}{ Siempre } & \multicolumn{2}{|c|}{$\begin{array}{c}\text { Casi } \\
\text { Siempre }\end{array}$} & \multicolumn{2}{|c|}{$\begin{array}{c}\text { Casi } \\
\text { Nunca }\end{array}$} & \multicolumn{2}{|c|}{ Nunca } & & \\
\hline & Dir & Doc & Dir & Doc & Dir & Doc & Dir & Doc & Dir & Doc \\
\hline Competencias Generales & 33,3 & 40,5 & 15,2 & 37,4 & 37,2 & 2,2 & 27,1 & 7,1 & 1,49 & 1,71 \\
\hline Competencias Espeáficas & 11,7 & 50,1 & 22,6 & 54,4 & 10,8 & 25,9 & 12,0 & 12,6 & 1,42 & 1,71 \\
\hline Promedio & 22,5 & 45,3 & 18,9 & 45,9 & 24,0 & 14,5 & 19,5 & 9,9 & 1,45 & 1,71 \\
\hline Media Directivos & & & & 1,45 & nada & Desarı & ollado & & & \\
\hline Media Docentes & & & & 1,71 & poco & Desarr & rollado & & & \\
\hline
\end{tabular}

Fuente: Elaborado por autores. 
situaron en la alternativa nunca, mientras que el $11,7 \%$, se situaron en la alternativa siempre, quedando un $10,8 \%$, en la alternativa casi nunca, evidencia que la medida de tendencia central para esta dimensión por parte de los directivos se situó en un 1.42, categorizándose según el baremo como poco nada desarrollado. Conforme a las respuestas suministradas por los docentes, se muestra que un $54,4 \%$, se situaron en la alternativa casi siempre, seguido de un $50,1 \%$, que se ubicaron en la alternativa siempre, mientras que el $25,9 \%$ se ubica en la alternativa casi nunca y solo el $12,6 \%$ se situaron en la alternativa casi nunca. Del mismo modo, que se puede comprobar que la medida de tendencia central para esta dimensión por parte de los sujetos sometidos al estudio se situó en un 1.71, categorizándose según el baremo como poco desarrollada.

Realizando el respectivo análisis de la variable competencias del docente deportivo, la evidencia que se muestra es que existen discrepancias en los resultados alcanzados en las opiniones de los directivos y docentes, obteniendo un valor promedio para los directivos de $24,0 \%$, se situados en la alternativa casi nunca, seguido de un $22,5 \%$, que se situaron en la alternativa siempre, mientras que el otro $19,5 \%$, se situaron en la alternativa nunca, quedando un $18,9 \%$, en la alternativa casi siempre.

Acorde a las respuestas provistas por los docentes, se muestra que un $45,9 \%$, se situaron en la alternativa casi siempre, seguido de un $45,3 \%$, que se ubicaron en la alternativa siempre, mientras que el
$14,5 \%$, se ubica en la alternativa casi nunca y solo el $9,9 \%$ se situaron en la alternativa nunca. De tal forma, se puede evidenciar que la medida de tendencia central para esta variable por parte de los sujetos sometidos al estudio se situó en un 1.45 , para los directivos y para los docentes en un 1.71, categorizándose según el baremo como nada desarrollado y poco desarrollada respectivamente.

En consideración a los resultados obtenidos, se muestran discrepantes de los postulados de Alles (2009), quien señala que las competencias son la capacidad para formar a otros tanto en conocimiento y capacidades. Implica un genuino esfuerzo para fomentar el aprendizaje a largo plazo y/o desarrollo de otros, más allá de su responsabilidad específica y cotidiana. En el mismo sentido, son más esenciales que las habilidades relacionadas con la inteligencia, tarea, o simplemente las credenciales, para predecir un desempeño elevado.

Por otra parte, Repetto et al (2006), señalan que el docente deportivo como persona integral requiere competencias básicas que lo identifiquen primero como persona y luego como profesional. Es necesario, entender que el docente deportivo va desde un aspecto fundamental que permite mantener una adecuada formación basada en las competencias, asimismo, los docentes deben formar a los estudiantes en competencias, esto significa, que lo formará para combinar, conocimientos, capacidades y comportamientos que se pueden utilizar e implementar 
directamente en un contexto de desempeño. En tal sentido, el resultado obtenido para la variable coincide con la investigación de Mendoza (2015), quien comprobó con su estudio que los directivos y docentes requieren mayor formación de sus competencias, de eficacia personal, administrativas $\mathrm{y}$ humano sociales. Toda vez que pretendan alcanzar el éxito organizacional al que están llamadas las instituciones educativas propiciadoras por excelencia de la calidad educativa que la sociedad actual tanto amerita.

La variable actitud de servicio que muestra la Tabla 4, refleja la actuación de esta variable por dimensión de la siguiente manera: la dimensión elementos básicos, se exhibe con un $38,6 \%$, para los directivos en la alternativa casi nunca, seguido de un $29,2 \%$, que se situaron en la alternativa nunca, mientras que el $15,2 \%$, se situaron en la alternativa siempre, quedando un $2,2 \%$, en la alternativa casi siempre, se puede evidenciar que la medida de tendencia central para esta dimensión por parte de los directivos se situó en un 1.48, categorizándose según el baremo como nada desarrollado. Conforme a las

Tabla 4.

Actitud de Servicio

\begin{tabular}{|c|c|c|c|c|c|c|c|c|c|c|}
\hline \multirow{3}{*}{ Dimensiones } & \multicolumn{8}{|c|}{ Alternativas (\%) } & & \\
\hline & \multicolumn{2}{|c|}{ Siempre } & \multicolumn{2}{|c|}{$\begin{array}{c}\text { Casi } \\
\text { Siempre }\end{array}$} & \multicolumn{2}{|c|}{ Casi Nunca } & \multicolumn{2}{|c|}{ Nunca } & \multicolumn{2}{|c|}{ Medias } \\
\hline & Dir & Doc & Dir & Doc & Dir & Doc & Dir & Doc & Dir & Doc \\
\hline Elementos Básicos & 15,2 & 45,2 & 2,2 & 40,5 & 38,6 & 21,8 & 29,2 & 7,1 & 1,48 & 2,01 \\
\hline Tipos de Actitud & 8,6 & 45,9 & 28,1 & 29,0 & 46,9 & 7,1 & 15,8 & 17,9 & 1,47 & 1,75 \\
\hline Promedio & 11,9 & 45,6 & 30,3 & 34,7 & 41,9 & 14,5 & 22,5 & 12,5 & 1,48 & 1,88 \\
\hline Media Directivos & \multicolumn{10}{|c|}{1,48 nada desarrollado } \\
\hline Media Docentes & \multicolumn{10}{|c|}{1,88 poco desarrollado } \\
\hline
\end{tabular}

Fuente: Calles y Bracho (2019). respuestas suministradas por los docentes, se muestra que un $40,2 \%$, se situaron en la alternativa siempre, seguido de un $40,5 \%$, que se ubicaron en la alternativa casi siempre, mientras que el $21,8 \%$ se ubica en la alternativa casi nunca y solo el $7,1 \%$ se situaron en la alternativa nunca. Es de destacar, que se puede evidenciar que la medida de tendencia central para esta por parte de los sujetos sometidos al estudio se situó en un 2.01, categorizándose según el baremo como poco desarrollado.

Seguidamente, los porcentajes logrados en la dimensión tipos de actitud, revela que un $46,9 \%$, de los directivos se situaron en la alternativa casi nunca, seguido de un $28,1 \%$, que se situaron en la alternativa casi siempre, mientras que el $15,8 \%$, se situaron en la alternativa nunca, quedando un $8,6 \%$, en la alternativa siempre, evidencia que la medida de tendencia central para esta situó en un 1.47, categorizándose según el baremo como poco nada desarrollado. Conforme a las respuestas proveídas por los docentes, se muestra que un $45,9 \%$, se situaron en la alternativa siempre, seguido de un $29,0 \%$, que se ubicaron en dimensión por parte de los directivos se 
la alternativa casi siempre, mientras que el $17,9 \%$ se ubica en la alternativa nunca y solo el 7,7\% se situaron en la alternativa casi nunca. Del mismo modo, que se puede comprobar que la medida de tendencia central para esta dimensión por parte de los sujetos sometidos al estudio se situó en un 1.75, categorizándose según el baremo como poco desarrollada.

Realizando el respectivo análisis de la variable actitud de servicio, la evidencia que se muestra es que existen discrepancias en los resultados alcanzados en las opiniones de los directivos y docentes, obteniendo un valor promedio para los directivos de $41,9 \%$, situados en la alternativa casi nunca, seguido de un $30,3 \%$, que se situaron en la alternativa casi siempre, mientras que el otro $22,5 \%$, se situaron en la alternativa nunca, quedando un $11,9 \%$, en la alternativa siempre. Acorde a las respuestas provistas por los docentes, se muestra que un $45,6 \%$, se situaron en la alternativa siempre, seguido de un $34,7 \%$, que se ubicaron en la alternativa casi siempre, mientras que el $14,5 \%$, se ubica en la alternativa casi nunca y solo el $12,5 \%$ se situaron en la alternativa nunca. De tal forma, se puede evidenciar que la medida de tendencia central para esta variable por parte de los sujetos sometidos al estudio se situó en un 1.48, para los directivos y para los docentes en un 1.88, categorizándose según el baremo como nada desarrollado y poco desarrollada respectivamente.

Los resultados antes descritos son discrepantes de los postulados de Campbell (1963, p. 96) quien afirma que una actitud de servicio del docente deportivo "es un síndrome de consistencia de respuestas hacia objetos sociales". Basado en lo anterior la disposición es una de las cualidades características de la actitud, por lo que se explica más por la vía procesual que por la vía del producto. Es necesario entender que la actitud de servicio permite que el docente deportivo desarrolle las actividades mostrando el interés y siendo promotor social de esas actividades deportivas.

Por otra parte, Dawes (1983), considera que la actitud de servicio es un afecto o disponibilidad para responder de cierta manera frente a un objeto $o$ fenómeno social que está relacionado con un componente valorativo. Una actitud de servicio permite que el docente muestre todos los conocimientos y competencias para desarrollar las actividades deportivas involucrando a los estudiantes y comunidades. Aunado a lo anterior, los resultados obtenidos por la variable coincide con la investigación de Rodríguez (2014), en la cual propuso analizar la actitud de servicio del docente ante la integración de Tecnologías de Información y la Comunicación, concluyendo que la actitud de servicio presentada por los docentes es indiferente ante la integración de las TIC, mostrando además que no están muy de acuerdo en la disposición de aprender para ser utilizada como herramienta de la enseñanza, aunque les resulte difícil comprender los conceptos relativos al uso del computador en clase.

Con el propósito de establecer la relación entre competencias del docente 
deportivo y su actitud de servicio, se efectuó una prueba conocida como correlación de Spearman (ver Tabla 5). Según Silva y Salinas (2007, p. 39), ' La correlación de Sperman se basa en reemplazar los valores originales, de ambas variables, por números enteros positivos, comenzando del 1 en adelante, que correspondan a su ordenamiento de menor a mayor magnitud (rangos)". Asimismo, Spearman, (1910) refiere que su coeficiente es una prueba no paramétrica que mide la asociación o interdependencia entre dos variables discretas medidas, al menos una de ellas, en escala ordinal.

Aplicada la fórmula de correlación de Rho Spearman de 0,74 a un nivel de significancia de 0.01 , lo cual indica que hay una relación positiva $y$ estadísticamente significativa entre las variables. Asimismo, para la discusión de los resultados, se elaboró un baremo previamente establecido que se construyó con base en los intervalos que ofrecen rango y categorías (ver Tabla 6). Este resultado se traduce en el hecho de que las competencias del docente deportivo se desarrollen y en la misma proporción será efectiva la actitud de servicio en Instituciones de Educación Primaria Bolivariana del Municipio mara del Estado Zulia.
Tabla 6

Baremo para la Interpretación del Coeficiente de Spearman

\begin{tabular}{cc}
\hline Rango & Categorías \\
\hline 0,81 a 1,00 & Muy alta \\
0,61 a 0,80 & Alta \\
0,41 a 0,60 & Moderada \\
0,21 a 0,40 & Baja \\
0,01 a 0,20 & Muy baja \\
\hline
\end{tabular}

Fuente: Calles y Bracho (2019).

\section{Conclusiones}

Es necesario que el docente asuma cuáles son sus competencias en el campo de la educación y demuestre disposición al trabajo donde es menester prestar toda colaboración a las instituciones educativas. Son necesarios docentes proactivos que realicen actividades de participación masiva donde se encuentren involucrados instituciones cercanas $\mathrm{o}$ lejanas a la de origen con la finalidad que las comunidades sean activas en el proceso educativo de sus hijos. Esa proactividad permitirá generar acciones de consolidacion de las actividades deportivas, así como de recreacion dentro de la institución educativa. Es necesario señalar que, en la actualidad, los docentes deportivos necesitan de incentivos para que sean proactivos, específicamente cumplan con la praxis pedagógica establecida como estructura. Por su parte, los docentes deportivos son los

Tabla 5.

Actitud de Servicio

\begin{tabular}{lcl}
\hline Variable & $\begin{array}{c}\text { Competencias del } \\
\text { Docente Deportivo }\end{array}$ & Actitud de Servicio \\
\hline
\end{tabular}

Competencias del Docente Deportivo

Actitud de Servicio

Correlación: 0.74

Fuente: Calles y Bracho (2019). 
encargados de llevar a cabo la recreación tomando en consideración su importancia en el desarrollo del estudiante, con la finalidad de mantener la salud física, mental y el equilibrio emocional a través de los juegos y movimientos corporales, los cuales permiten que cada persona se mantenga activa. Con ello el docente deportivo pasa a ser un promotor social llevando a las comunidades actividades deportivas y de recreación, lo cual ayudan en el crecimiento de las instituciones educativas y la salud de comunidades.

Los docentes deportivos tienen la responsabilidad de la salud mental y física no sólo de los estudiantes, sino también de la comunidad, en otras palabras, deben integrarlos para el mejoramiento de la calidad educativa. En muchas ocasiones la necesidad de manejo de competencias hace que existan actitudes de servicio basadas en la apatía de las personas, ya sean de las comunidades o simplemente de los estudiantes, lo que genera conflictos constantes.

En consencuencia, los docentes deportivos necesitan mantener activas las competencias educativas, físicas $\mathrm{y}$ gerenciales, asegurando la salud física y mental de todos los involucrados y generando el equilibrio emocional. El docente también tiene competencias específicas que se encuentran representadas en la praxis pedagógica, es decir la manera de llevar el conocimiento, promotor social que no es más que la manera de organizar la actividades llevándolas a las comunidades e involucrándolos en el proceso educativo, en el área deportiva. Esa actitud de servicio de los docentes, afectada por la necesidad de vocación y del compromiso de abordar los temas deportivos y que sólo se encuentran en el medio por la parte económica origina que su rendimiento sea deficiente. Todo esto llevaría a que el docente tuviese una actitud negativa del servicio, no sólo en las instituciones educativas, sino también en las comunidades, específicamente cuando se trata de su involucramiento en las actividades deportivas y de recreación.

\section{Referencias}

Alles, M. (2009). Desempeño por competencias: evaluación de $360^{\circ}$ (8va ed.). Buenos Aires, Argentina: Granica S.A.

Alles, M. (2009). Selección por competencias. Edición ilustrada (4ta ed.). Buenos Aires, Argentina: Ediciones Granica S.A.

Álvarez, C. (1985). Preparación Física del Fútbol Basada en el Atletismo. Madrid, España: S.L. Gymnos.

Arias, F.G. (2006). El Proyecto de Investigación. Introducción a la metodología científica (5ta ed.). Caracas, Venezuela: Episteme, C.A.

Bavaresco, A. (2008). Las técnicas de la investigación. Manual para la elaboración de tesis, monografias, informes (6ta ed.). Maracaibo, Venezuela: Universidad Del Zulia.

Bohlander, G.W., Snell, S.A., y Morris, S. S. (2017). Administración de Recursos Humanos (17ava ed.). México DF, México: Cengage Learning.

Bracho, K. (2019). Competencias Investigativas del Docente para el Fortalecimiento de su Praxis Pedagógica. Revista Educare, 
23(1), 188-201.

Campbell, D.T. (1963). Social attitudes and other acquired behavioral dispositions. En S. Koch (Ed.), Psychology: A study of a Science (Vol. 6, pp 94-172). New York, NY: McGraw-Hill.

Chávez, N. (2007). Introducción a la investigación educativa (4ta ed). Maracaibo, Venezuela: Talleres Gráfica González, C.A.

Chiavenato, I. (2006). Introducción a la teoría general de la administración (7ma ed). DF- México, México: Mc-Graw Hill Interamericana Editores S.A.

Dawes, M. (1983). Fundamentos y técnicas de medición de actitudes. México: Editorial Limusa.

Edvinsson, L. y Malone, M. (2007). El capital intelectual cómo identificar y calcular el valor de los recursos intangibles de su empresa. Barcelona, España: Ediciones Gestión 2000.

González, Z. (2010). Estrategias motivacionales para el fortalecimiento de la organización deportiva. Especialista en Administración Deportiva. Tesis de Especialista. Universidad Del Zulia, Venezuela.

Hernández, R., Fernández, C., y Baptista, P. (2008). Metodología de la Investigación (5ta ed.) México. D.F., México: McGraw - Hill Interamericana S.A.

Isaza, M. (2010). Educación Fisica. Parte I: Conceptos básicos. Ministerio de Educación Nacional, República de Colombia.

López, N., Montes, J. y Vázquez, C. (2007). Cómo gestionar la innovación en las pymes. Catálogo general. La Coruña, España: Netbiblo.

Maduro, N. (2013). Segundo Plan Socialista de Desarrollo Económico y Social de la
Nación, 2013-2019. República Bolivariana de Venezuela.

Mendoza, R. (2015). Competencias del director y docente en Instituciones Educativas de Colombia y Venezuela. Tesis de Maestria. Universidad Dr. Rafael Belloso Chacín.

Morales, C. (2007). La Educación Bolivariana una Perspectiva Social. Universidad Central de Venezuela. Facultad de Humanidades y Educación. División de Postgrado. Caracas - Venezuela.

Myers, D. (2005). Psicología (7ma ed.). Madrid, España: Editorial Médica Panamericana.

Olson, J. y Zanna, M. (1993). Attitudes and attitude change. Annual Review of Psychology, 44(1), 117-154.

Pérez, E. y Camejo, D. (2009). Síntesis gráfica de supervisión educativa (aula abierta). Madrid, España: Editorial La Muralla.

Ramírez, J. (2002). Planificar en Educación Física. Barcelona, España: INDE

Reinfeld, B., y Rojas, T. (2005). Reflexiones sobre la apatía. Archivos venezolanos de Psiquiatría y Neurología, 51(105), 19-25.

Repetto, E., Beltrán, S., Garay-Gordovil, A, y Pena, M. (2006). Validación del inventario de competencias socioemocionales -importancia y presencia-" (ICS-I; ICS-P) en estudiantes de ciclos formativos y de universidad. Revista Española de Orientación y Psicopedagogía, 17(2), 213-223.

Robbins, S. y DeCenzo, D. (2009). Supervisión (5ta ed.). México: Pearson Educación.

Rodríguez, M. (2014). Actitud de servicio del docente universitario ante la integración de las tecnologías de información y comunicación. Tesis de Maestría. Universidad Dr. Rafael Belloso Chacín. Venezuela. 
Sanz, M. (2010). Competencias cognitivas en Educación Superior. Madrid, España: Narcea Ediciones.

Silva, C. y Salinas, M. (2007). Modelos de Regresión y Correlación. Revista Ciencia y Trabajo, (22), 185-189.

Spearman, C. (1910). Correlation calculated from faulty data. British Journal of Psychology, 3(2), 271-295.

Summers, D. (2006). Administración de la Calidad. México: Pearson Educación

Tobón, S. (2005). Formación basada en competencias: Pensamiento complejo, diseño curricular y didáctica. Bogotá, Colombia: ECOE.

Torres, J. (2009). Gestión directiva en la aplicabilidad de la educación intercultural bilingüe en instituciones educativas. Tesis de Maestria. Universidad Dr. Rafael Belloso Chacín. Venezuela.

Vezub, L. y Alliaud, A. (2012). El acompañamiento pedagógico como estrategia de apoyo y desarrollo profesional de los docentes noveles. Aportes conceptuales y operativos para un programa de apoyo a los docentes principiantes de Uruguay. Organización de Estados Iberoamericanos.

Vieytes, R. (2004). Metodología de la Investigación en Organizaciones, Mercado y Sociedad. Epistemología y Técnicas. Buenos Aires, Argentina: Editorial de las Ciencias.

PODIUM No. 36, Diciembre 2019, pp. 55-72

(C) Universidad Espíritu Santo - UEES

ISSN: 1390-5473 e-ISSN: 2588-0969 
